\title{
EVALUACIÓN DE LA VIDA ÚTIL POSTCOSECHA DE PERA (PACKHAM'S TRIUMPH) MEDIANTE RECUBRIMIENTO CON CERA DE CARNAUBA Y CERA DE ABEJA
}

"EVALUATION OF THE POSSIBLE LIFE OF PEAR (PACKHAM'S TRIUMPH) THROUGH COATING WITH CARNAUBA WAX AND BEE WAX"

Hugo Rafael García Miranda ${ }^{1}$ Hebert Quintín Zúñiga Vargas ${ }^{1}$ Danny Daniel Carhuaz Valdez ${ }^{1,2}$

Información del artículo: Recibido: 11/03/2019. Aceptado: 20/06/2019

1 Ingeniero Agroindustrial

${ }^{2}$ Docente Facultad de Ingeniería en Universidad Privada de Tacna 


\section{Resumen}

El estudio de la aplicación de recubrimientos sobre la pera es muy escaso, por ese motivo la investigación tuvo como objetivo "Evaluar el efecto que tiene la cera de abeja y cera de carnauba sobre la vida útil postcosecha de pera (Packham's triumph)", la evaluación se llevó acabo en la Universidad Privada de Tacna, laboratorio de análisis de productos agroindustriales. Para ello se empleó cera de carnauba y cera abeja. Mediante experimentos previos se formuló tratamientos con cera de abeja (TCA), con cera de carnauba (TCC) y un tratamiento mezcla (TM, "cera de abeja y cera de carnauba") donde resultó que el recubrimiento con mayor estabilidad fue mezcla de $40 \%$ cera de abeja y 60 \% cera de carnauba, para efectos de análisis se identificó los parámetros necesarios a medir, como son los sólidos solubles totales (SST), la acidez titulable, el pH, el peso, el color, el $\mathrm{CO}_{2}$ y la Firmeza. Se concluye que el tratamiento mezcla (TM, "cera de abeja $40 \%$ y cera de carnauba60 \%") tuvo mejores resultados, aumentado la vida útil en cinco y seis días adicionales, los datos obtenido a los 17 días de estudio fueron, sólidos solubles totales $\left(11,3^{\circ} \mathrm{brix}\right)$, la acidez titulable $(0,0415 \%)$, el pH $(4,98)$, el peso $(13,48 \%)$, el color (café verdoso), el $\mathrm{CO}_{2}$ (27352.59 mg. $\mathrm{CO}_{2} / \mathrm{Kg}$.h) y la Firmeza $(2,54 \mathrm{~N})$.

Palabras Claves: $\mathrm{CO}_{2}$, Carnauba, Pera, Cera de abejas.

\section{Abstract}

The study of the application of coatings on the pear is very scarce, for this reason the research aimed to "Evaluate the effect of beeswax and carnauba wax on the post-harvest life of pear (Packham's triumph)" The evaluation was carried out at the Universidad Privada de Tacna, Laboratory for the analysis of agroindustrial products. For this, carnauba wax and beeswax were used. Through previous experiments, treatments with beeswax (TCA), with carnauba wax (TCC) and a mixed treatment (TM, "beeswax and carnauba wax") were formulated, where it was found that the coating with greater fuel stability mix $40 \%$ beeswax and $60 \%$ carnauba wax, for analysis purposes the parameters needed to measure were identified, such as total soluble solids (SST), titratable acidity, pH, weight, color, $\mathrm{CO}_{-} 2$ and Firmness Concluding that the mixture treatment (TM, "beeswax 40\% and carnauba wax 60\%") had better results, increased the useful life in an additional five and six days, the data obtained at 17 days of study were, strongly Total soluble $\left(11.3^{\circ}\right.$ brix), titratable acidity $(0.0415 \%), \mathrm{pH}(4.98)$, weight $(13.48 \%)$, color (greenish brown), CO_2 (27352.59 mg. CO_2 /Kg.h) and Firmness (2.54 N).

Key Words: CO2, Carnauba, Pear, Bees

\section{introducción}

La vida útil postcosecha de pera (Packham's triumph) mediante recubrimiento con cera de carnauba y cera de abeja, prolonga su vida útil. La tendencia actual de la industria de alimentos va de la mano con los cambios de estilo de vida de las personas. El desperdicio de productos vegetales y frutas se debe a inadecuadas prácticas en el momento de la cosecha, y las derivadas 
del acondicionamiento y tratamientos posteriores que inciden de manera significativa sobre las características de calidad, afectando su comercialización y distribución en los mercados. Respecto a la pera, su problemática post cosecha no es diferente, existes problemas relacionados a perdida de humedad/peso, tasa de respiración, madurez fisiológica generado por el mismo fruto. Ya que son productos climatéricos tienden a madurar más rápido debido a que siguen respirando después de ser cosechados. Se han realizado estudios sobre el uso de recubrimiento biodegradable a partir de polisacáridos, proteínas y lípidos con el propósito de determinar su efectividad como barrera en la transferencia de masa y gases. La cera de carnauba y la de abeja son lípidos simples. El uso de cera de carnauba y cera de abeja como recubriendo cuya función es formar barrera en la superficie del fruto, podría ayudar a modular el intercambio de gases, perdida de humedad, controlan el arrugamiento, reducen la senescencia, aportan brillo a la piel del fruto e incrementan el periodo de comercialización con la finalidad de alargar la vida útil del fruto.

Fernando et al. (2015) investigó la evaluación de un recubrimiento comestible a base de proteína de lactosuero y cera de abeja. Observó la reducción en un 35,49\% de pérdida de peso del fruto con respecto a la de la muestra testigo. Acosta et al. (2013) evaluó un recubrimiento comestible para la conservación postcosecha del tomate de árbol cyphomandra betacea Cav. Sendt; teniendo como resultado el incremento de la vida útil en un $25 \%$ más. Maccapa (2015) Investigó el efecto del uso de un recubrimiento biodegradable a base de aislado de proteína de suero sobre la vida útil de la chirimoya (annona cherimola), fresa (fragaria vesca) y pera (pyrus communis). Se determinó que en la Fresa y Chirimoya la aplicación del recubrimiento Comestible no fue optima en ninguna de sus formulaciones, las frutas con y sin recubrimientos mostraron similares cambios en los parámetros fisicoquímicos. Asimismo, se determinó que en la pera la aplicación de aislado de proteína de suero - Glicerol - Cera de Abeja como recubrimiento permitió el desarrollo de un efectivo método de conservación que prolongó la vida útil y no afectó a los parámetros fisicoquímicos. Rodríguez (2017) investigó el estudio de la aplicación de un recubrimiento comestible a base de quitosano al yacón dando como resultado 5 días de vida útil a diferencia del grupo testigo. Costa et al. (2012) estudió los cambios de la composición de los cítricos después del recubrimiento con la cera de carnauba en condiciones de comercialización loca $\left(22+2{ }^{\circ} \mathrm{C}, 60 \mathrm{HR}\right)$, dando como resultado final un incremento de 5 días respecto a la prueba testigo. Cáceres et al. (2003) investigó la influencia del encerado en la calidad de la mandarina mostrando que la concentración de $2 \%$ de cera de carnauba y candelilla fue eficaz para el mantenimiento de la calidad demostrando un incremento de 7 días. Así mismo Márquez et al. (2007) investigó el efecto de la aplicación de un recubrimiento comestible a base de gelatina sobre la calidad de fresa almacenadas en refrigeración, observando una firmeza de $79 \%$, pérdida de peso del $54 \%$ con respecto a las fresas sin recubrimiento alargando la vida útil en un 50\%. Hernández et al. (2011) estudió el efecto del recubrimiento a base de almidón de yuca sobre la maduración del tomate dando como resultado una pérdida de peso del $54 \%$, y una firmeza de $2.54 \mathrm{~N}$ hasta el día 22, el recubrimiento con un 4 \% de almidón fue el más efectivo en la conservación de tomate de mesa. Miranda et al. (2014) utilizando el almidón de yuca a una concentración del $4 \%$, las papayas almacenadas a temperatura ambiente $22{ }^{\circ} \mathrm{C}$ y una humedad relativa del $85 \%$ por un periodo de 9 días, se observó un $33 \%$ de pérdida de peso. Ccosi y Riveros (2014) investigaron sobre la evaluación de recubrimientos comestibles a base de mucilago de 
chía (salvia hispánica I.) sobre la conservación post cosecha de palta (persea americana mill.) Presentaron menores promedios de pérdidas de peso $(10,92 \%)$, seguidos de aquellos recubiertos con el tratamiento RC-01 (1,01\% MG y 0,20\% EC) y RC-09. (1,75\% MG y 0,50 \% EC). Espinoza (2017) investigó sobre el Quitosano y almidón como recubrimiento biodegradable para prolongar la vida útil en palta, y concluye que los mejores tratamientos evaluados a los 15 días presentaron de 8,01 a 8,4 de Grados Brix, 13,54 a 14,85 \% de contenido de aceite, 30,2 a $32,96 \%$ de materia seca, 67,04 a $69,8 \%$ de humedad, 7,38 a 7,7 de pH, 0,10 a 0,16 de acidez titulable. García et al. (2017) y su estudio a base de quitosano, almidón de yuca y aceite esencial de canela para conservar pera (Pyrus communis L. cv. "Bosc"). Los tratamientos que contienen quitosano presentaron mayor reducción microbiológica durante los 20 días de almacenamiento. Villamón et al. (2018) evaluaron el uso de diferentes recubrimientos comestibles para extender la vida útil de manzana y pera y evalúa el efecto de 14 recubrimientos formulados con diferentes biopolímeros. Los recubrimientos desarrollados tuvieron un mayor efecto reduciendo la pérdida de peso en manzana que en pera.

En la industria de los alimentos tiene de gran importancia el empaquetado, para el tema de seguridad alimentaria y vida útil del producto, el efecto que tendrán los envases sobre los alimentos dependerá de las características de acuerdo con los materiales que se usaran para elaborarlas, de ello dependerá la calidad en cuestión de textura, color, sabor, olor, etc. De los productos alimentarios (Peula, 2017).

\section{OBJETIVOS}

- Evaluar la mejor formulación para la mezcla de cera de carnauba y cera de abeja utilizada como recubrimiento en la pera

- Evaluar la vida útil de la pera (Packham's triumph) con el uso de cera de carnauba y cera de abeja

- Evaluar la tasa respiratoria, la pérdida fisiológica del peso de la pera, el pH y los cambios en el color de la pera recubierta del mejor tratamiento y una muestra testigo en la etapa de postcosecha.

\section{METODOLOGÍA}

Investigación experimental en una primera etapa; y en una segunda etapa tendrá un carácter cuasi experimental. La muestra es por conveniencia. En la figura 1 se presenta las principales etapas para el acordonamiento de la materia prima. En la figura 2. Se presenta las principales etapas para la elaboración del recubrimiento donde se tiene como componente principal la cera de abeja y cera de carnauba.

Evaluación de la eficacia del recubrimiento aplicada en los frutos: mediante el programa estadístico statgraphic se podrá determinar el mejor recubrimiento sobre la fruta. La determinación del cambio de acidez titulable a lo largo del tiempo se evaluará en peras recubiertas y no cubiertas, según la metodología AOAC 950.07 (1984) adaptada por Parra y Sánchez (1998). Para el análisis Ph se usó la metodología A.O.A.C. 935.15 (1980) adaptado por Gilbert et al. (2013). Se determinaron los sólidos Solubles Totales (SST) expresado en Brix", con el fin de obtener el contenido de azucares de la muestra según Rosario (2016). La pérdida de peso se midió mediante la diferencia entre el peso del fruto al iniciar el período de 
almacenamiento y el peso del fruto al terminar este período. Los resultados fueron expresados como porcentaje de pérdida de peso (\%PP) según Vásquez Lara y Vidal López (2011). La firmeza es uno de los métodos fisicoquímicos que mejor se relación con el estado de madurez del fruto según Brezmes (1999). La determinación de $\mathrm{CO}_{2}$ se hizo según Cartagena (2016). Para las mediciones se utilizó el sensor K33 BLG, diseñado para medir el dióxido de carbono, temperatura y \% de humedad relativa. Para el análisis de color se usó el aplicativo "identificador de color" del programa Color Compañy - Analyzer \& Converter.,

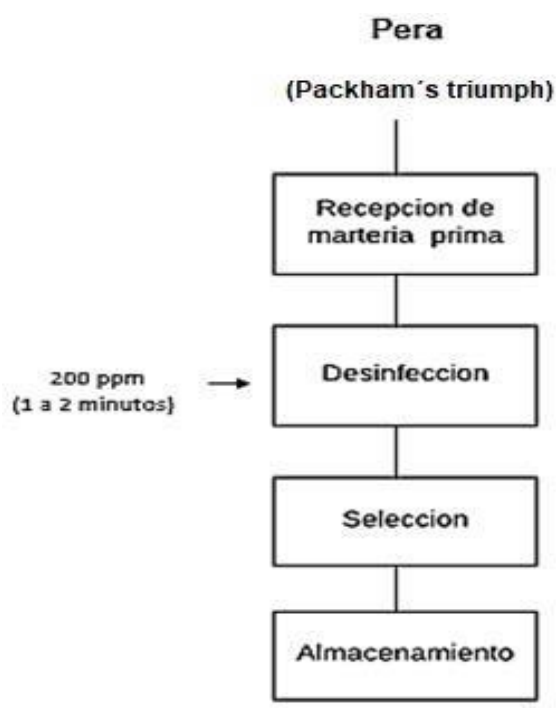

Figura 1. Representación gráfica acondicionamiento de la pera

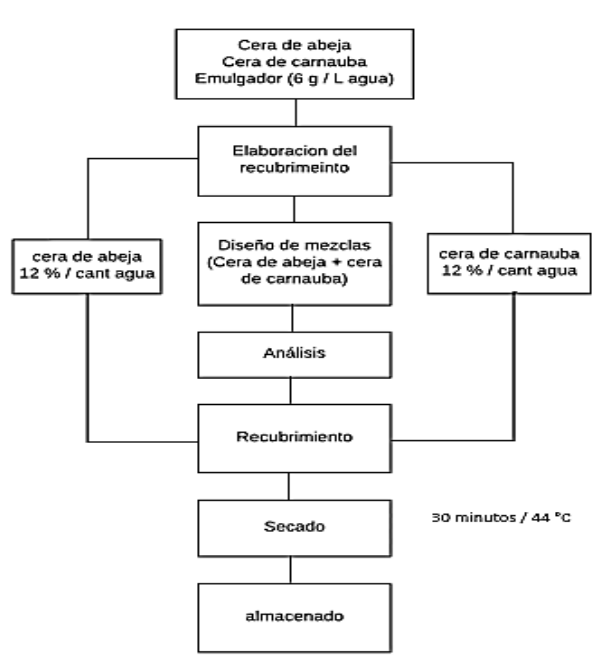

Figura 2. Representación gráfica del diseño cuasi experimental, elaboración propia.

\section{RESULTADOS}

Se aplicó el mismo diseño de mezclas utilizado en la tesis para la elaboración de un recubrimiento por Condori (2017), el cual empleo un diseño experimental completamente al azar utilizando un valor mínimo de $40 \%$, utilizando el programa estadístico statgraphics centurión xvii, el cual dio como resultado cinco tratamientos experimentales (Tabla 1). Se realizaron cinco tratamientos, tratamiento 1 (T1) constituye de $50 \%$ de cera de abeja y $50 \%$ de cera de carnauba, tratamiento 2 (T2) se compone de $40 \%$ de cera de abeja y $60 \%$ de cera de carnauba, tratamiento 3 (T3) se compone de $60 \%$ de cera de abeja y $40 \%$ cera de carnauba, tratamiento 4 (T4) constituye de $45 \%$ de cera de abeja y $55 \%$ cera de carnauba, y el tratamiento 5 (T5) de $55 \%$ de cera de abeja y $45 \%$ de cera de carnauba.

Tabla 1

Tratamientos según el diseño de mezclas

\begin{tabular}{|c|c|c|}
\hline Tratamiento & Cera de abeja (\%) & Cera de carnauba (\%) \\
\hline $\mathrm{T} 1$ & 50 & 50 \\
\hline $\mathrm{T} 2$ & 40 & 60 \\
\hline T3 & 60 & 40 \\
\hline T4 & 45 & 55 \\
\hline T5 & 55 & 45 \\
\hline
\end{tabular}


En la tabla 2 se muestra un resumen de los valores hallados expresados en porcentaje, los análisis que se tomaron son: prueba de centrifugado y estabilidad de emulsión por calor, donde el tratamiento 2 al obtener mayor porcentaje de estabilidad a comparación de los demás tratamientos fue elegida para su aplicación en la pera.

Tabla 2.

Análisis de los Tratamientos según sus

porcentajes de composición.

\begin{tabular}{lcccc}
\hline $\begin{array}{l}\text { Tratamien } \\
\text { to }\end{array}$ & $\begin{array}{c}\text { Cera de } \\
\text { abeja (\%) }\end{array}$ & $\begin{array}{c}\text { Cera de } \\
\text { carnauba (\%) }\end{array}$ & PC (\%) & PE (\%) \\
\hline T1 & 50 & 50 & 0,495 & 0,62 \\
T2 & 40 & 60 & 0,16 & 0,34 \\
T3 & 60 & 40 & 0,383 & 0,43 \\
T4 & 45 & 55 & 0,295 & 0,45 \\
T5 & 55 & 45 & 1,875 & 1,46 \\
\hline
\end{tabular}

En la tabla 3 se muestra los valores obtenido de la prueba de centrifugado por triplicado dando como resultados un promedio de cada valor, estos resultados serán de suma importancia para determinar cuál de los tratamientos tiene mejor estabilidad. Existe una relación estadísticamente significativa $(p=0,0004)$ entre los tratamientos.

Tabla 3

Resultado de la toma de muestras de la prueba de centrifugado

\begin{tabular}{lcccc}
\hline Pruebas & \multicolumn{2}{c}{ Medidas (\%) } & \multicolumn{2}{c}{ Promedio (\%) } \\
\hline T1 & 0,55 & 0,44 & 0,485 & 0,495 \\
T2 & 0,15 & 0,17 & 0,16 & 0,16 \\
T3 & 0,307 & 0,46 & 0,413 & 0,3835 \\
T4 & 0,17 & 0,42 & 0,263 & 0,295 \\
T5 & 2,05 & 1,7 & 1,875 & 1,875 \\
\hline
\end{tabular}

En la tabla 4 se muestra los valores obtenido de la prueba de estabilidad por triplicado dando como resultados un promedio de cada valor, estos resultados serán de suma importancia para determinar cuál del tratamiento tiene mejor estabilidad. Existe una relación estadísticamente significativa ( $p=0,0037)$ entre los tratamientos, con un nivel de confianza del $95 \%$.

Tabla 4

Resultado de la toma de muestras de la prueba de estabilidad

\begin{tabular}{lcccc}
\hline Pruebas & \multicolumn{2}{c}{ Medición (\%) } & \multicolumn{2}{c}{ Promedio (\%) } \\
\hline T1 & 0,66 & 0,59 & 0,61 & 0,62 \\
T2 & 0,3 & 0,38 & 0,34 & 0,34 \\
T3 & 0,41 & 0,46 & 0,42 & 0,43 \\
T4 & 0,64 & 0,26 & 0,45 & 0,45 \\
T5 & 1,32 & 1,6 & 1,46 & 1,46 \\
\hline
\end{tabular}

En la Figura 1 se observa el comportamiento de la Acidez del tratamiento testigo (TO) evaluado durante 14 días, el comportamiento de T0 tiende a disminuir

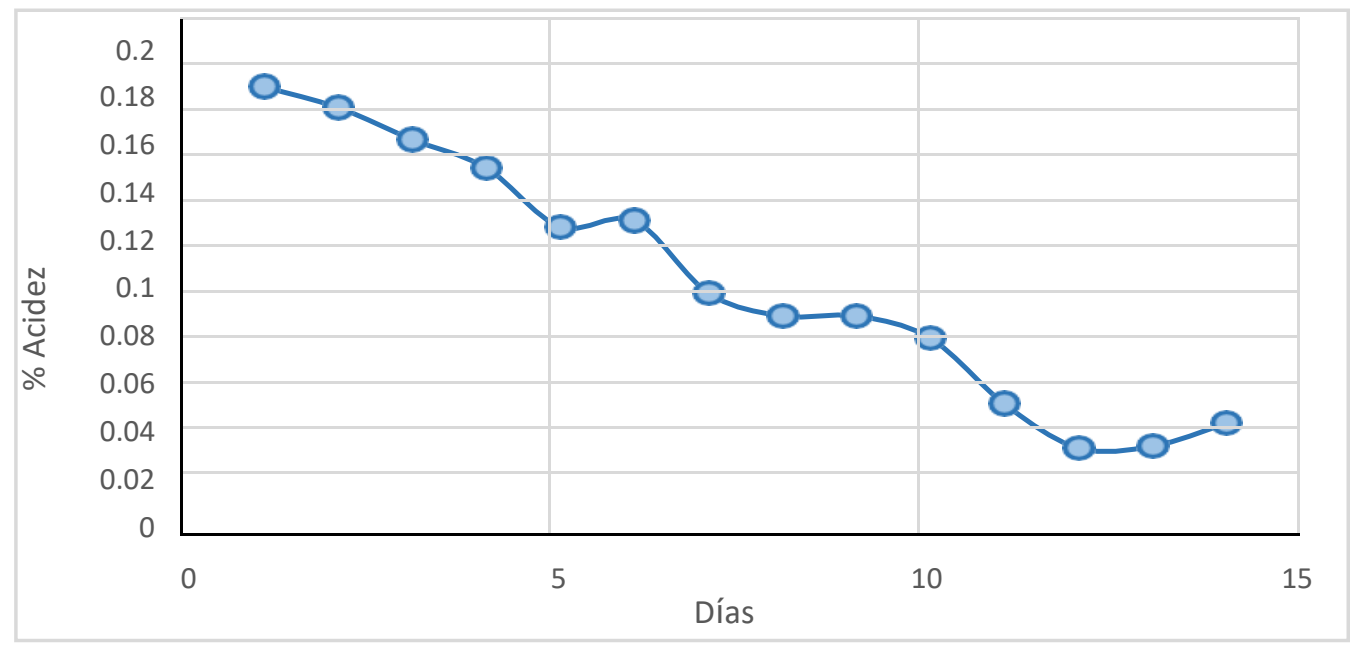


Figura 1 Análisis de Acidez. Comportamiento del tratamiento testigo (TO)

En la figura 2 se observa el comportamiento de los cuatro tratamientos los cuales tienden a disminuir con el tiempo

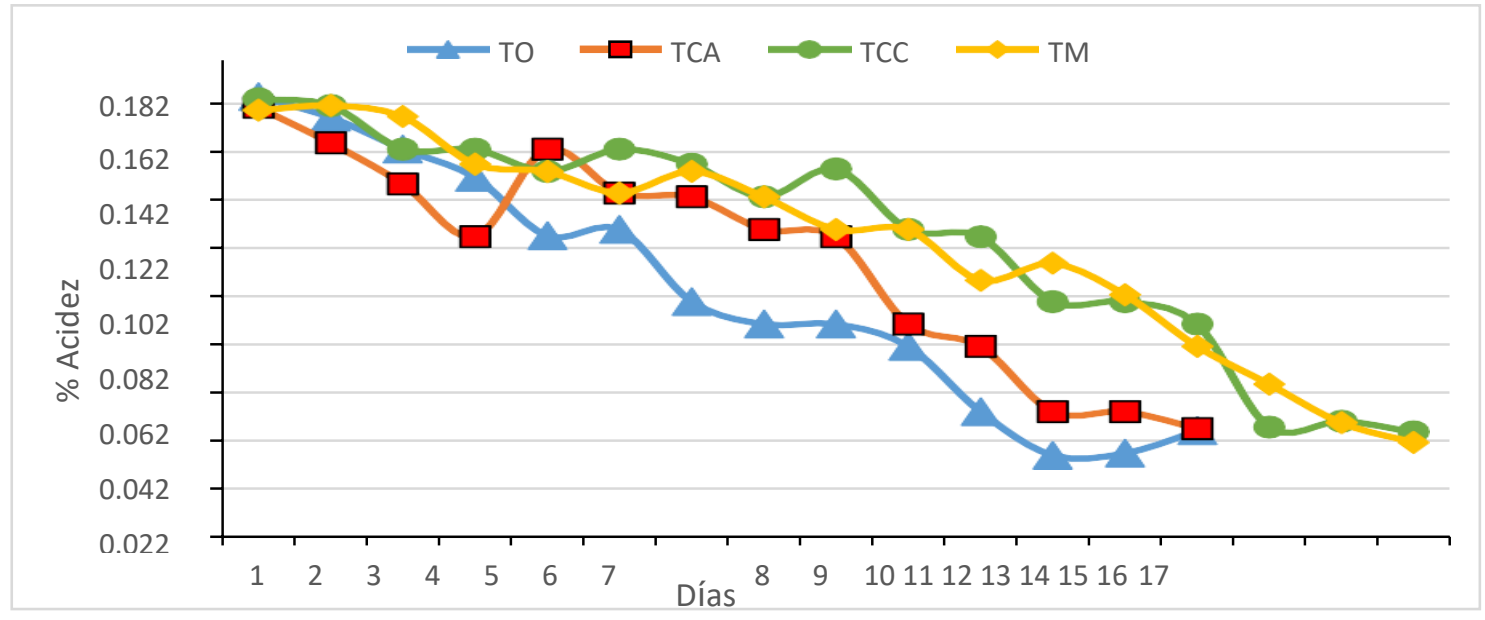

Figura 2 Análisis de Acidez. Comportamiento de la Acidez en los tratamientos T0 = tratamiento testigo; TCA = tratamiento cera de abeja; TCC = tratamiento cera de carnauba; TM = tratamiento mezcla (cera de abeja y cera de carnauba).

Los ácidos orgánicos se encuentran circulando en los tejidos vegetales tras la recolección, y tienden a disminuir en todo el periodo de pos cosecha hasta la etapa de senescencia. La mayor parte de esta pérdida se debe a su oxidación en el metabolismo respiratorio, reacción que se traduce como un incremento de cociente de respiración (Fennema et al., 2008). No existe diferencia significativa ( $p=0,5812)$ entre los tratamientos.

Las variaciones que puede presentar las frutas en su $\mathrm{pH}$ va a depender del tipo fruta, el estado de madurez, condiciones agronómicas y operaciones post cosecha a las que has sido sometidas (López y Mercado, 2006). En la Figura 3 se observa el comportamiento del porcentaje de $\mathrm{pH}$ de los tratamientos

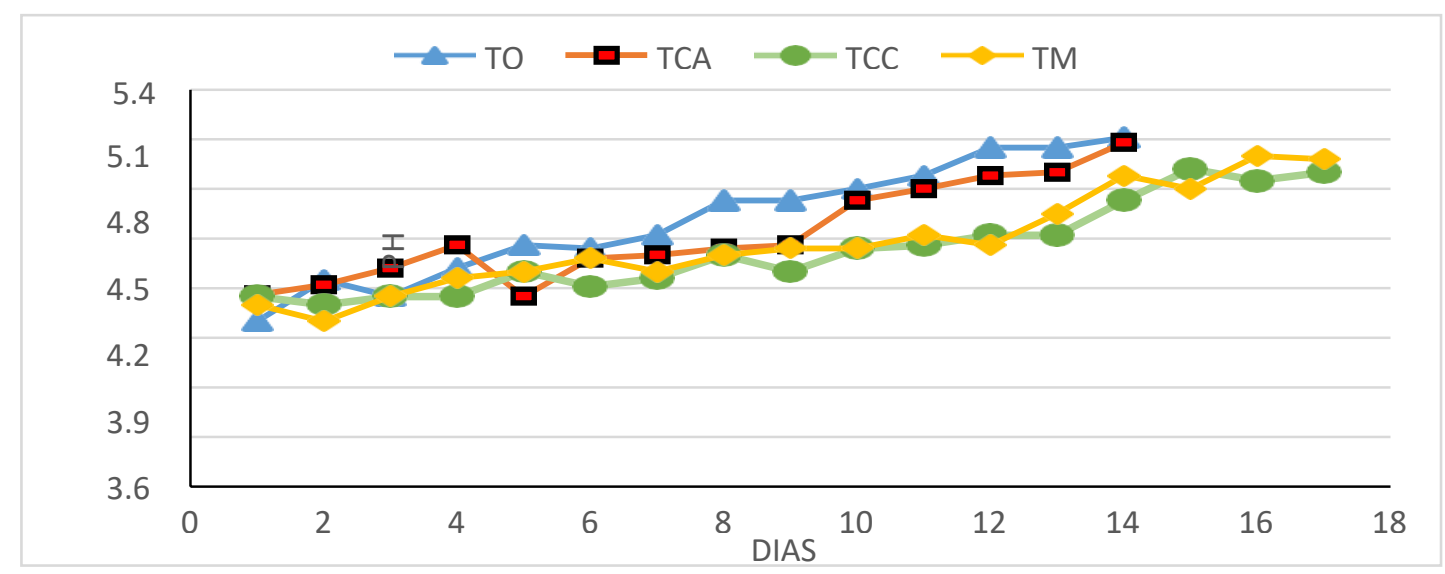

Figura 3. Análisis de pH. Comportamiento del pH en lostratamientos TO = tratamiento testigo; TCA = tratamiento cera de abeja; TCC = tratamiento cera de carnauba; TM = tratamiento mezcla (cera de abeja y cera de carnauba).

Se observa el incremento en el $\mathrm{pH}$ de la pera debido a que los ácidos orgánicos de reserva presentes en las vacuolas de las células, para luego transformarse en azúcares que son utilizados como fuente de energía, lo que genera la disminución de la acidez y el aumento del pH 
(López C, 2006 citado en Hernández J, 2013). No existe diferencia significativa $(p=0,4410)$ entre los tratamientos.

En la figura 4 se observa el comportamiento de los Solidos Solubles Totales que va en aumento con el paso del tiempo. No existe diferencia significativa $(p=0,4034)$ entre los tratamientos, con un nivel de confianza del $95 \%$

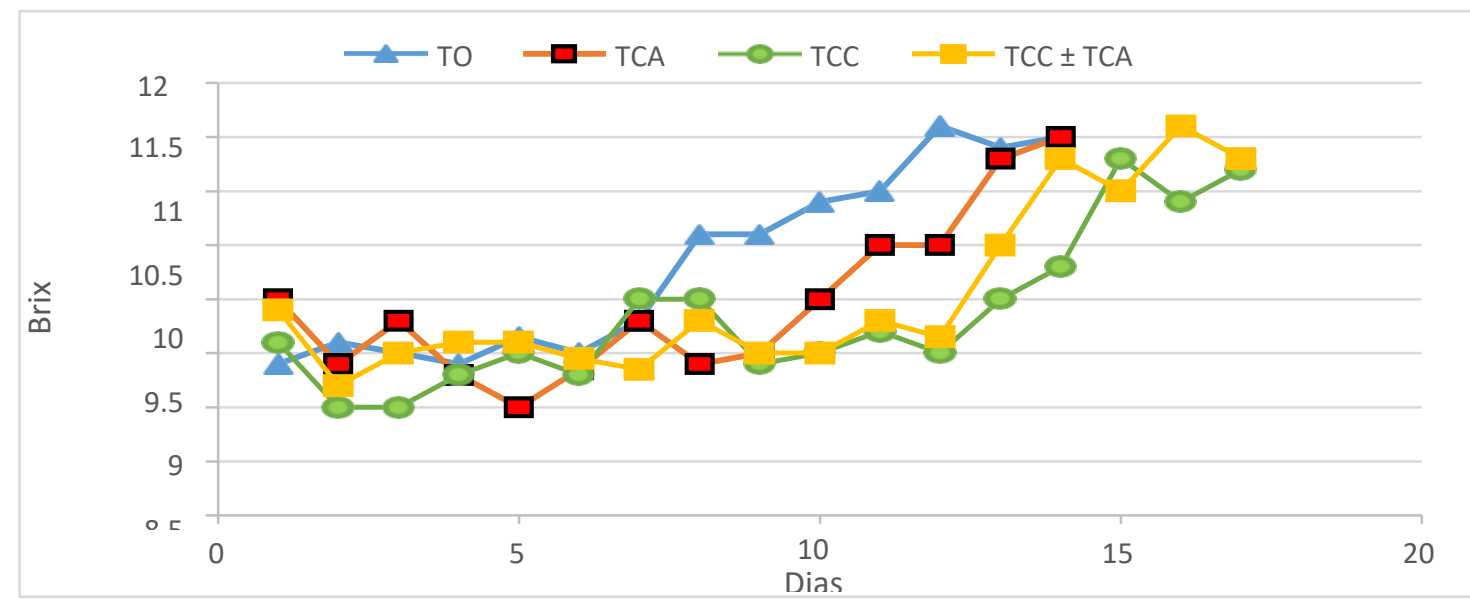

Figura 4. Análisis de Solidos Solubles Totales. Comportamiento de los Solidos Solubles Totales en los tratamientos $\mathrm{TO}=$ tratamiento testigo; TCA = tratamiento cera de abeja; TCC = tratamiento cera de carnauba; TM = tratamiento mezcla (cera de abeja y cera de carnauba).

El contenido de agua en las frutas compone del 90 al $95 \%$ del peso total y este a su vez tiene gran influencia sobre la calidad del fruto, es debido a que incluso la pérdida más pequeña de agua $(<1 \%)$ puede originar cambios fisiológicos el cual generará un impacto negativo reduciendo su vida útil (Damodaran, 2008). La fuerza que impulsa la pérdida de agua en el gradiente de concentración entre el vapor de agua en el interior de los espacios intercelulares del producto y el vapor de agua del entorno en el que se encuentra (Moreno y Flores, 2017).

En la figura 5 se observa el comportamiento de la pérdida de peso que va en aumento con el paso del tiempo. El efecto neto de la transpiración es una pérdida de agua del producto cosechado, que no puede ser reemplazada. La velocidad con que se pierde esta apara será un factor determinante en la vida de postcosecha del producto. La pérdida de agua causa una disminución significativa del peso y a medida que avanza, disminuye la apariencia y elasticidad del producto perdiendo su turgencia, es decir, se vuelve blando y marchito. (Moreno y Flores, 2017)

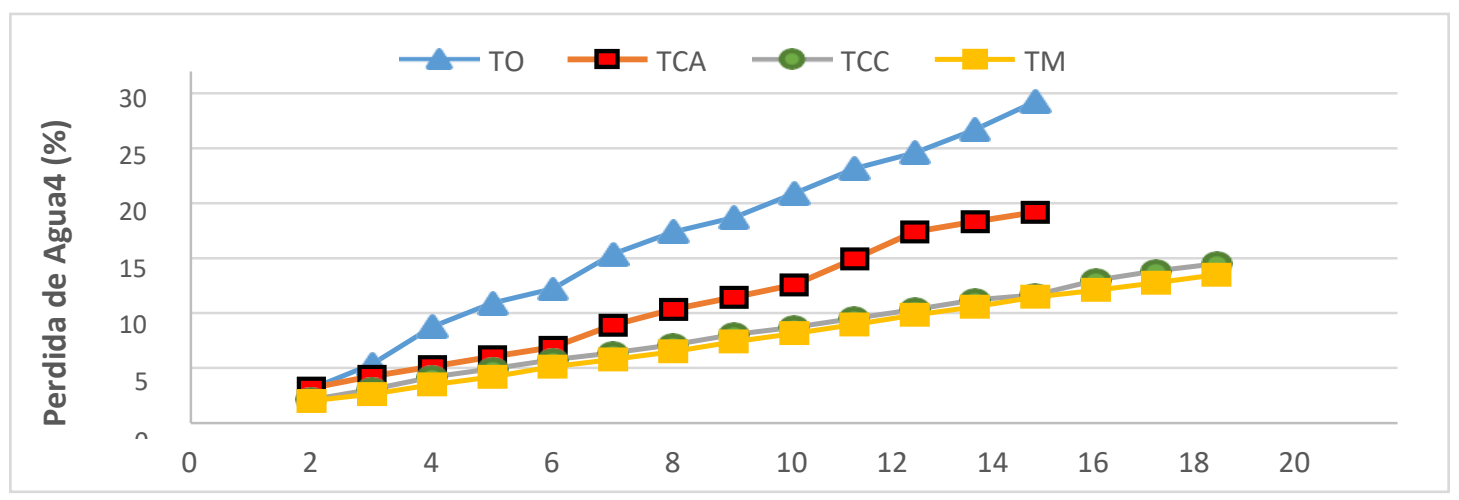

Figura 5. Análisis de Pérdida de peso expresada en porcentaje en los tratamientos T0 = tratamiento testigo; TCA = tratamiento cera de abeja; TCC = tratamiento cera de carnauba; TM = tratamiento mezcla (cera de abeja y cera de carnauba). Existe diferencia significativa $(p=0,0002)$ entre los tratamientos, con un nivel de confianza del $95 \%$ 
En la figura 6 se observa el comportamiento de la Firmeza que disminuye con el paso del tiempo

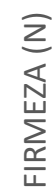

Ingeniería investiga Vol. 1 (1) Julio - diciembre 2019. 


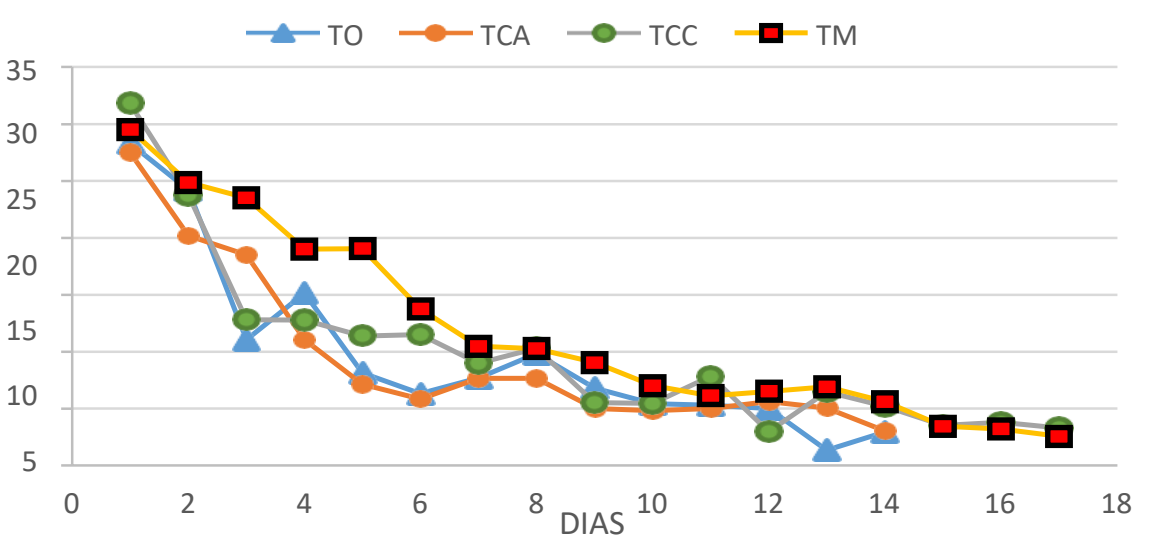

Figura 6. Análisis de Firmeza. Pérdida de agua expresada en porcentaje en los tratamientos T0 = tratamiento testigo; $\mathrm{TCA}=$ tratamiento cera de abeja; $\mathrm{TCC}=$ tratamiento cera de carnauba; $\mathrm{TM}=$ tratamiento mezcla (cera de abeja y cera de carnauba).

No existe diferencia significativa $(p=0,8326)$ entre los tratamientos, con un nivel de confianza del $95 \%$.

En figura 7 se observa el comportamiento de la tasa de respiración que va en aumento con el paso del tiempo. El recubrimiento incrementa desordenes asociados con unaalta concentración de $\mathrm{CO}_{2}$ (Ben-Yehoshua, 1969). Existe una diferencia estadísticamente significativa entre las medias de las 4 variables con un nivel del $95.0 \%$ de confianza.

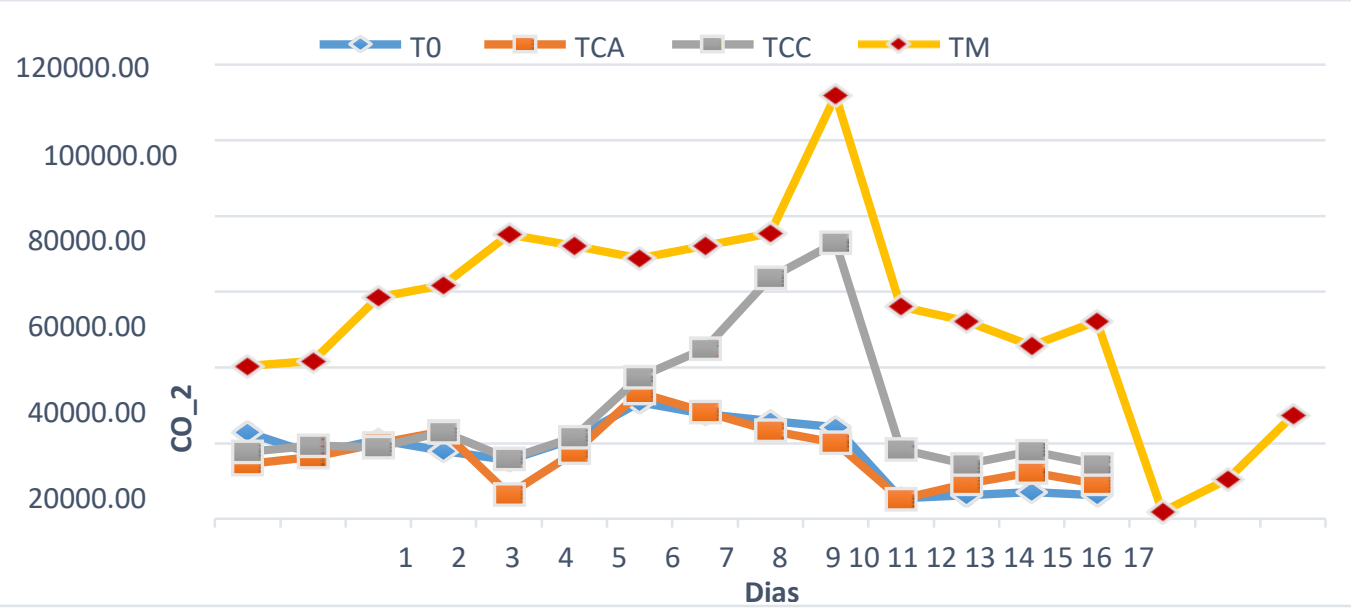

Figura 7. Análisis de $\mathrm{CO}_{2}$. Comportamiento de la Tasa de Respiración de los tratamientos, $\mathrm{TO}=$ tratamiento testigo; TCA = tratamiento cera de abeja; TCC = tratamiento cera de carnauba; TM = tratamiento mezcla (cera de abeja y cera de carnauba).

Tabla 5

Análisis de varianza de $\mathbf{C O}_{2}$ evaluada en los tratamientos

\begin{tabular}{lccccc}
\hline \multicolumn{1}{c}{ Fuente } & Suma de Cuadrados & Gl & Cuadrado Medio & Razón-F & Valor-P \\
\hline Entre grupos & $1,44672 \mathrm{E} 10$ & 3 & $4,82238 \mathrm{E} 9$ & 15,17 & 0,0000 \\
Intra grupos & $1,74821 \mathrm{E} 10$ & 55 & $3,17856 \mathrm{E} 8$ & & \\
Total (Corr.) & $3,19492 \mathrm{E} 10$ & 58 & & &
\end{tabular}

Nota: $S C$ = Suma de Cuadrados; Gl= grados de libertad; CM = Cuadrado medio; Razón-F=Distribución Fisher, Valor- $P=$ Significancia 
En la tabla 5, se evalúa la Prueba Múltiple de Rango con la finalidad de determinar cuáles medias son significativamente diferentes de otras. La mitad inferior de la salida muestra las diferencias estimadas entre cada par de medias. No existen diferencias estadísticamente significativas entre aquellos niveles que compartan una misma columna de X's.

Se observa en la tabla 6 el cambio de color del tratamiento testigo iniciando con un color Sycamore (\#958c2c), el día 5 se observa un ligero cambio Olive Green (\#a4a348), el día 10 el cambio de color es notorio pasando a Tussok (\#b79746), el día 14 el color sufre un cambio drástico leather (\#8f6848). El color de la fruta testigo (TO) es aceptable los 5 primeros días, ligeramente aceptable hasta el día 10 y posteriormente tiende a un color no aceptable a simple vista. Los colores aparecen con sus componentes primarias de rojo, verde y azul. Los valores de $R, G, y$ B se encuentran a lo largo de tres ejes, lo que se refleja en datos es la intensidad de cada color. Todos los valores de R, G y B están en el intervalo $[0,1]$. En el caso de imágenes digitales los valores de R, G y B son números enteros y van de 0 a 255, lo cual permite generar 16777216 colores (Pérez, 2009).

Tabla 6

Colores del tratamiento testigo (T0) por cada 5 días

\begin{tabular}{c|ccc|c}
\hline Día & R & G & B & Color \\
\hline 1 & 149 & 140 & 44 & \#958c2c \\
5 & 164 & 163 & 72 & \#a4a348 \\
10 & 183 & 151 & 70 & \#b79746 \\
14 & 143 & 104 & 72 & \#8f6848 \\
\hline
\end{tabular}

Nota: La intensidad de color toma valores de rojo $(R)$, verde $(G)$ y azul (B)

Asimismo, se observa cambio de color del tratamiento con cera de abeja en un periodo de 14 días, iniciando con un color Sycamore (\#9a962e), el día 5 se mantiene el color Sycamore \#8f8b35), el día 10 el cambio de color es notorio pasando a Husk (\#c1a95a), el día 14 el color sufre un cambio drástico a Driftwood (\#a27c4b). El color de la fruta (TCA) es aceptable los 5 primeros días, ligeramente aceptable hasta el día 10 y posteriormente tiende a un color no aceptable a simple vista. Con cera de carnauba por cada 5 días, se observa el cambio de color iniciando con un color Sycamore (\#999b39), el día 5 se mantiene el color Sycamore (\#9e983c), el día 10 mantiene el color con ligero cambio en la tonalidad a Sycamore (\#9e8c44), el día 14 el color sufre un cambio drástico a Spice (\#6f4c39). El color de la fruta (TCA) es aceptable los 5 primeros días, incluso hasta el día 10 y posteriormente tiende a un color no aceptable a simple vista.

Los colores del tratamiento Mezcla (Cera de abeja y carnauba) por cada 5 días, se observa el cambio de color del tratamiento Mezcla (Cera de abeja y carnauba) iniciando con un color Trendy Green (\#99a036), el día 5 se observa un cambio mínimo a Sycamore (\#8b903a), el día 10 no hay cambio Sycamore (\#878b41), el día 14 el color se mantiene Sycamore (\#888630) y finalmente el día 17 se observa un ligero cambio a Costa del sol (\#5e5d28). El color de las peras con recubrimiento de carnauba y cera de abeja (TM) es aceptable los 14 primeros días, ligeramente aceptable hasta el día 17 a simple vista.

La vida útil de la pera dependerá de los parámetros de $\mathrm{pH}$ (4.04-4.90) establecidos por (Parra et al., 1998), los cuales se mostrará en la figura 19. Sé que el comportamiento del tratamiento que obtuvo mayor vida útil (TM) tiene un promedio de 15 a 16 días y el tratamiento que tiene una menor vida útil (TO) tiende a durar 11 días.

En la figura 8 vemos el comportamiento de los tratamientos TM y TO durante su evaluación, la caída de $\mathrm{CO}_{2}$ en los días TO (10 a 11) y TM (14 a 15) entrando a su etapa de senescencia, eso indica que es apto para su vida comercial. 


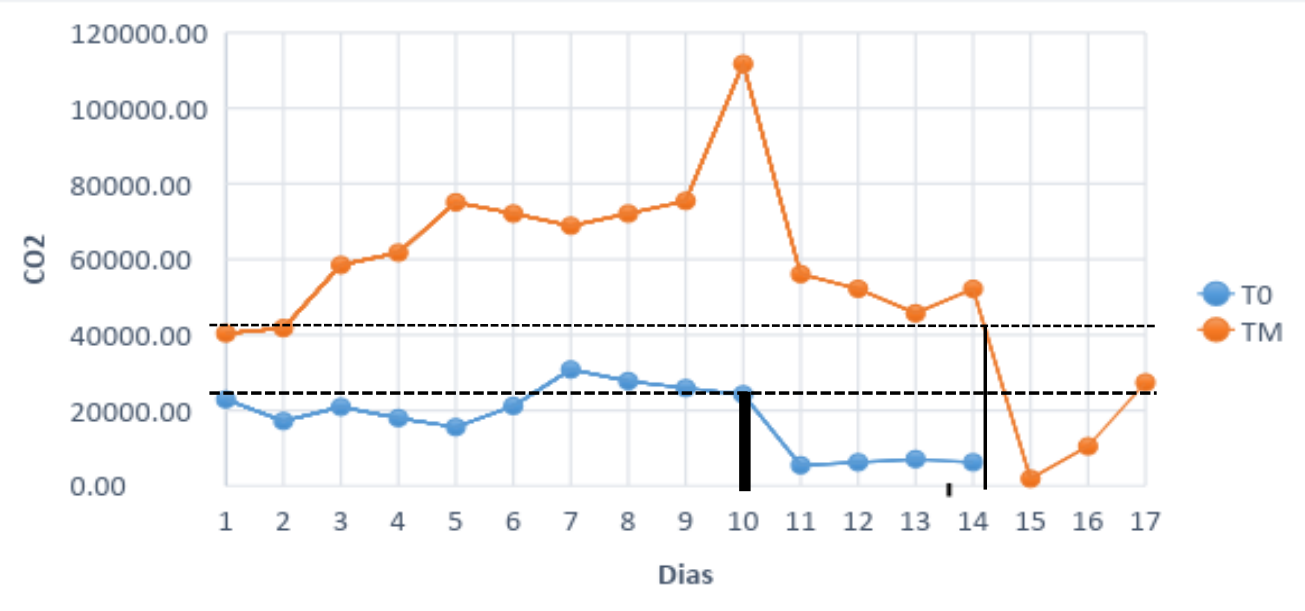

Figura 8. Determinación del $\mathrm{CO}_{2}$ de los tratamientos; Tratamiento testigo (TO) y tratamiento mezcla TM (cera de abeja más cera de carnauba).

El comportamiento de la firmeza del tratamiento (TM) en el primer día fue de $29.5 \mathrm{~N} / \mathrm{m} 2$ y el último día fue de $2.56 \mathrm{~N} / \mathrm{m} 2$, este comportamiento de debe a que las sustancias que le proporcionan la turgencia al fruto (Pectina y Proto pectina) se transforman en ácidos péptidos solubles en agua y otras sustancias, produciendo ablandamiento en las frutas (Hernández et al., 2006).

En la Figura 9 se observa el comportamiento que tienen la firmeza y la acidez durante 17 días del mejor tratamiento TM (cera de carnauba y cera de abeja).

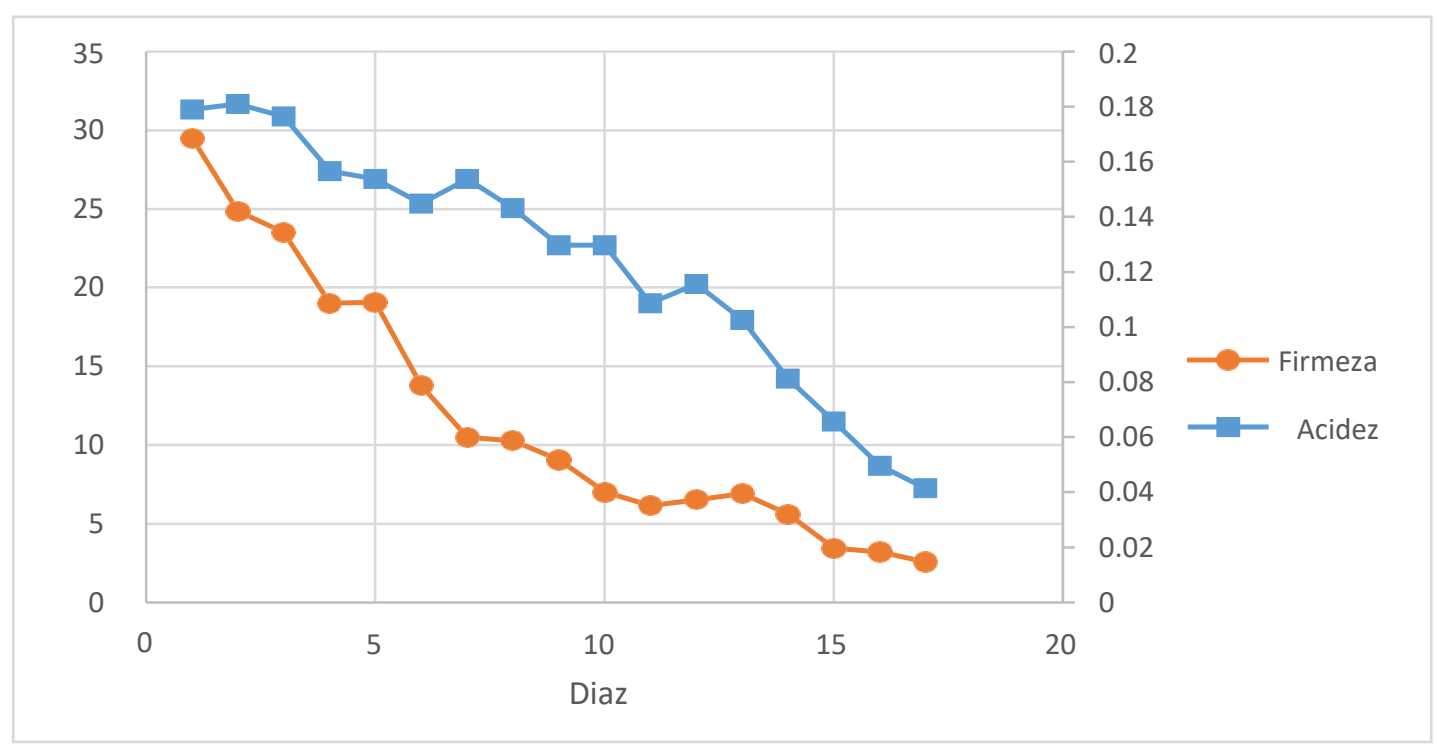

Figura 9. Comportamiento de la acides titulable y de firmeza de la pera variedad Packham's triumph

El contenido de acidez es un factor importante en la vida útil de las frutas, este está relacionado la capacidad de proliferación microbiana, valores bajos de acidez permitirá una vida más corta de las frutas (Ruiz y Rodríguez, 2014). En la figura 10 se muestra la relación de pH y la acidez, en cuanto la Acidez disminuye el pH aumenta, debido a la liberación de ácido orgánico en la respiración de las frutas y a su incremento de sólidos solubles 


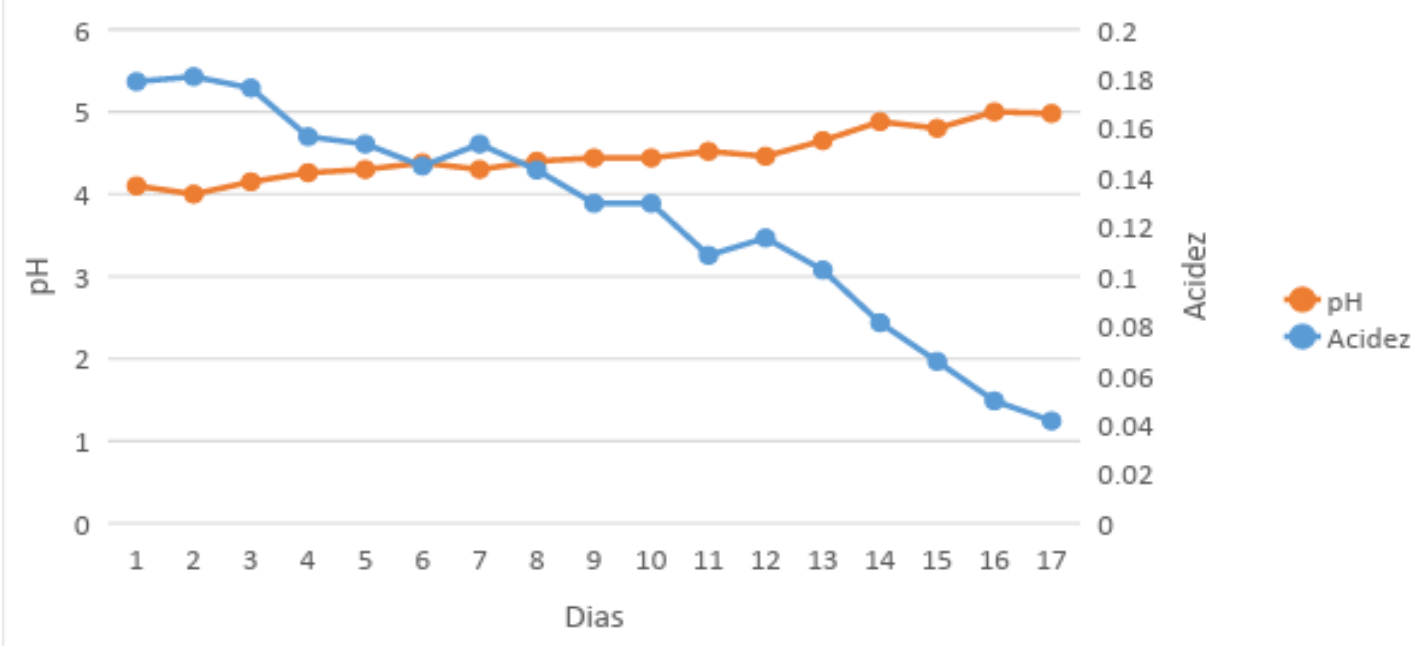

Figura 10. Comportamiento del pH y Acidez durante 17 días de la pera variedad Packham's triumph.

\section{DISCUSIÓN}

La proporción utilizada del recubrimiento fue del $12 \%$ en solución acuosa, este resultado se obtuvo del promedio de las investigaciones realizadas por (Fernando et al., 2015) quien evaluó un recubrimiento a base de proteína de sueroy cera de abeja sobre la uchuva donde determino la mejor proporción del recubrimiento la cual fue de $12,95 \%$, asimismo Quintero (2012) en el estudio de la formulación de un recubrimiento comestible para frutas cítricas tiene un proporción de $12 \%$ ya que este disminuye la pérdida de peso de las fruta.Para finalizar (Acosta et al., 2013) en la elaboración y evaluación de un recubrimiento comestible para la conservación postcosecha del tomate de árbol utilizó 10,6 \% de recubrimiento sobre solución acuosa. La investigación determinó que el mejor es el tratamiento con cera de abaja y ceras de carnauba (TM) que corresponde a la mezcla compuesta por $40 \%$ cera de abeja y $60 \%$ cera de carnauba aumento la vida útil de la pera de 5 días de diferencia con la muestra testigo (TO), obteniendo resultados similares de Maccapa (2015) quien obtuvo un aumento de 6 días en el estudio de efecto de la aplicación de un recubrimiento biodegradable a base de aislado de proteína de suero sobre la vida útil de la chirimoya y fresa, también Villegas y Albarracín (2016) investigaron el efecto de un recubrimiento comestibles a base de hidroxipropil metilcelulosa con la inclusión de cera de abejas sobre la vida útil de la mora de castilla donde obtienen un incremento de 10 días de diferencia con la muestra testigo y el mejor tratamiento, así mismo Rodríguez (2017) obtuvo un incremento de 5 días en el estudio de efecto del quitosano como revestimiento comestible en yacón mínimamente procesado para aumentar su vida útil. El tratamiento (TCA) cera de abeja no fue significativo, porque no hubo diferencia con el tratamiento testigo (TO), quienes tuvieron 12 días de vida útil, obteniendo valores inferiores a los de Villegas y Albarracín (2016) quienes evaluaron la aplicación de un recubrimiento comestible sobre la vida útil de la mora de castilla, mantenido las características fisiológicas intactas de la mora durante 15 días usando como recubrimiento hidroxipropil meticulosa y cera de abeja a una temperatura de $4 \stackrel{\text { a }}{\mathrm{C}}$.

El tratamiento TCA (cera de abeja) no fue significativo, porque no hubo diferencia con el tratamiento testigo (TO), quienes tuvieron 11 días de vida útil según los parámetros establecido por Parra (1998). El tratamiento TCC (cera de carnauba) aumento la vida útil de la pera por 4 días de diferencia con la muestra testigo T0, obteniéndose menores que (Costa et al., 2012) quien establece que el aumento de la fresa con cera de carnauba a $22^{\circ} \mathrm{C}$ fuera de 5 días, 
también establece Cáceres et al. (2003) que aumentó 7 días con el uso de cera de carnauba sobre la mandarina variedad Clemenules. El análisis de pérdida de peso el tratamiento TM (cera de abeja y cera de carnauba) fue el que tuvo menor pérdida de peso con un valor de $11,483 \%$ hasta los 14 días y el tratamiento testigo fue de $29,261 \%$, evitando una pérdida de líquido del $17,778 \%$ con respecto a la prueba testigo, así mismo (Marquez et al., 2007) determinó en el estudio de la aplicación de gelatina sobre la calidad de la fresa que a los 10 días el mejor tratamiento tuvo una pérdida de peso que de $13 \%$, y el tratamiento testigo fue de $20 \%$, evitando una pérdida de líquido del $7 \%$. También (Hernández et al,2011), en el estudio del efecto del recubrimiento a base de almidón de yuca modificado sobre la maduración del tomate, obtuvo valores del mejor tratamiento que a los 14 días la pérdida de peso fue de 8,33\%, y el tratamiento testigo fue de $11,11 \%$, evitando una pérdida de líquido del $2,78 \% \%$ con respecto a la prueba testigo. Para finalizar (Miranda et al., 2014), estudio el efecto de dos recubrimientos sobre la calidad de la papaya donde obtienen resultados hasta los 9 días, donde resulta que el mejor tratamiento con almidón fue de $9,54 \%$, y el tratamiento testigo fue de $12,75 \%$, evitando así un pérdida de peso del 3,21\%.con respecto al tratamiento testigo.

El análisis de firmeza el tratamiento TM (cera de abeja y cera de carnauba) fue el que mantuvo mayor firmeza de rompimiento a los 14 día obtuvo una firmeza de $5,6 \mathrm{~N}$ y el tratamiento testigo fue de 2,54 $\mathrm{N}$ evitando una pérdida de firmeza del 54,64 \%, Asimismo (Miranda et al., 2014) estudio el efecto de dos recubrimientos sobre la calidad de la papaya durante 9 días donde el mejor tratamiento evito una pérdida del $38 \%$ en relación al tratamiento testigo, también (Hernández et al.,2011) en el estudio del efecto del recubrimiento a base de almidón de yuca modificado sobre la maduración del tomate obtuvo valores que a los 17 días el mejor tratamiento tuvo valor de 9,54 $\mathrm{N}$ evitando así una pérdida del 67,57 \%. Para finalizar (Estrada et al., 2015), en el estudio del efecto de recubrimiento protectores sobre la calidad del mango en postcosecha, tuvo un rendimiento de $54 \%$ siento este menor a nuestros resultados. De los datos recopilados en la medición de $\mathrm{CO}_{2}$, se observa mejores resultados en el tratamiento mezcla (cera de abeja y carnauba) con valores más alto de respiración, manteniéndose por encima de los demás tratamientos tanto en la disminución como en el incremento de la tasa respiratoria, este comportamiento lo explica Avena-Bustillos, Krochta y Saltveir (1997), donde indican que las propiedades del fruto dependerán del tiempo de material utilizado como recubrimiento, este mecanismo es crear una barrera física a los gases, reduciendo la disponibilidad de $\mathrm{O} 2$ e incrementan la concentración de $\mathrm{CO} 2$. Así mismo BenYehoshua (1969). Según el tipo de recubrimiento aplicado en la fruta puede modificar su atmosfera interna e incrementar desordenes asociados con una alta concentración de $\mathrm{CO} 2 \mathrm{o}$ una baja de O2. El valor más alto registrado fue el día 10 con un valor $111759,11 \mathrm{mg}$. CO2/Kg.h., climaterio alcanzado para todos los tratamientos y a su vez término de la vida útil con excepción del tratamiento mezcla (cera de abeja y carnauba) prolongando su vida útil por 4 días, tomando el día 14 un valor de 52165,9 mg. CO2/Kg.h. Del mismo modo Figueroa (2013) mediante el uso de almidón de yuca como de recubrimiento en frutos de mango cv. Tommy atkins, consiguió extender el pico climatérico de estos frutos en dos días adicionales. En este punto Gonzáles (2013). Confirma patrones de respiración elevados con tendencia ascendente y presencia de picos climatéricos en el arazá. Respecto a la producción de etileno Cardozo (2012) observó que en las frutas de soursop estudiadas crecía durante la postcosecha, con picos sobresalientes en los días 4 y 6 , siendo este último el más grande, con una producción de $186,9 \mathrm{ml} . \mathrm{CO}_{2} / \mathrm{Kg}$.h. Las frutas alcanzaron su producción máxima de etileno el día 8 después de la cosecha, al anotar 133,2 $\mathrm{ml}$. $\mathrm{CO}_{2} / \mathrm{Kg}$.h.

Por la comparación de las imágenes realizadas a lo largo del experimento, en los tratamientos testigo y con cera de abeja se observa tendencia a un color amarillento, por el contrario, el tratamiento con cera de carnauba y la mezcla (cera de carnauba y de abeja) mantiene el color verde oscureciendo levemente. El mejor resultado de color verde característico de la pera se observa en el tratamiento mezcla (cera de carnauba y de abeja) por lo 
que podemos concluir que el recubrimiento ayuda a mantener el color durante más tiempo. En otros recubrimientos con quitosano, menciona Han et al. (2004) "El recubriente para frambuesa retrasa cambios en el color, acidez titulable y pH, además mejora la textura de la fruta". Para Gómez (2011) los recubrimientos comestibles en mango influyen en los parámetros de calidad de la fruta incluyendo el color, la firmeza, las concentraciones de sólidos solubles, acidez total, ácido ascórbico, carotenoides totales, ácidos grasos volátiles. En el mismo sentido Falguera et al. (2011) determina que la apariencia es elatributo de mayor importancia en alimentos frescos y mínimamente procesados, con aspectos primarios considerados como tamaño y uniformidad de color, brillantez, y ausencia de defectos de contorno o aspecto de la piel.

Se recomienda hacer estudios de vida útil con la aplicación de la mejor formulación (60 $\%$ cera de carnauba y $40 \%$ cera de abeja) almacenados en estado de refrigeración en la pera. Evitar exponer el fruto a largos tiempos de secado con aire caliente, ya que esto acelera su deterioro. Se sugiere que en estudios posteriores se realice la evaluación sensorial por medio de paneles entrenados con el fin de emitir un concepto en la aceptación de la pera. En una futura investigación con recubrimiento de cera de carnauba y abeja se recomienda evaluar la tasa de respiración de etileno.

\section{REFERENCIAS}

Aguirre L., Roció Y. (2011). Efecto del ácido oleico sobre las propiedades de transferencia de masa de películas biodegradables de quitosano. Toluca - México.

Andrea D., Zulma G., Leonardo F. (2005). Informe de laboratorio recepción de materia prima, análisis de control de calidad de frutas y verduras. CEDEAGRO.

Arévalo A., Azucena P., Laínez A. (2018). Formulación y caracterización de una biopeliculas comestible elaborada a partir de almidón de Sorgo y Yuca. El Salvador.

Aguilar M., M. A. (2013). Propiedades físicas y mecánicas de películas biodegradables y su empleo en el recubrimiento de frutos de aguacate. México.

Avena-Bustillos, R., Krochta, J. y Saltveit, E. (1997). Water vapour resistance of red delicious apples and celery sticks coated with edible caseinate- acetylated monoglyceride films. Journal of Food Science, 62, 351-354.

Brezmes LL. (1999). Diseño de una nariz electrónica para la determinación no destructiva del grado de maduración de la fruta. Técnicas de control de calidad en fruta.

Márquez J., Yepes D., Sánchez L., y Osorio A. (2017). Cambios fisicoquímicos del aguacate (Persea americana Mill. cv. "Hass") en postcosecha para dos municipios de Antioquia. Colombia.

Cabrera, L. (2003). Aplicación de métodos combinados para el control del desarrollo del pardeamiento enzimático en pera (variedad Blanquilla) mínimamente procesada. Valencia.

Cardozo, C. J. M., Lozano, V. V., Betancur, D. P. Y., Velásquez, H. J. C., \& Valenzuela, J. R. C. (2012). Caracterización Fisiológica y Físico-Química del Fruto de la Guanábana (Annona muricata L. cv. Elita). Revista Facultad Nacional de Agronomía Medellín, 65(1), 64776486.

Cartagena, R. (2016). Implementación de un sistema automatizado de monitoreo y control de la tasa respiratoria en postcosecha de frutas. Tacna - Perú.

Castillo G. (2004). Desarrollo de métodos cromatográficos para la determinación de gibelinas y auxinas en caldos de fermentación. Ciudad de la Habana.

Cáceres I., Martínez M., y Navarro P (2003). Influencia del encerado en la calidad de la mandarina "Clemenules" procedentes de sistemas de producción integrada. Revista Iberoamericana de tecnología postcosecha. México.

Ciro Arias, Julio Toledo. (2007). Manual de Manejo de Postcosecha de Frutas Tropicales. FAO.

Ccosi V., y Riveros R. evaluación de recubrimientos comestibles a base de mucílago de chía 
(salvia hispánica I.) sobre la conservación postcosecha de palta (persea americana mill.) variedad hass, Ayacucho - Perú.

Costa M., Machado C., y Batista M. (2012). Aplicacation of carnauba-based wax maintains postharvest quality of 'Ortanique' tangor. Ciencia y Tecnología de alimentos.

Estrada M., Padilla R., y Márquez C. (2015). Efecto de recubrimiento protectores sobre la calidad del mango (Mangifera indica L.) en poscosecha. Colombia.

David Fernando, Liseth Yurani, Johana Carolna y Oswaldo Osorio. (2015). Evaluación de un recubrimiento comestible a base de proteínas de lactosuero y cera de abeja sobre la calidad fisicoquímica de uchuva (Physalis peruviana L.). Nariño-Colombia.

Damoradan, S. (2008). Fennema química de los alimentos. ACRIBIA.

FAO. (1992). Manual para el control de calidad de los alimentos. Alimentación y nutrición.

Falguera V., Quintero. P., Jiménez A., Muñoz J. A., Ibarz A. 2011. Edible films and coatings: Structures, active function and trends in their use. Trends in Food Science and Technology 22:292-303.

Fernández V., Bautista B., Ocampo R., y Falcón R. (2015). Películas y recubrimientos comestibles: una alternativa favorable en la conservación postcosecha de frutas y hortalizas. En Revistas Ciencias Tecnológicas Agropecuarias 94-105.

Figueroa, J. A., Salcedo, J. G., y Narváez, G. J. (2013). Efecto de recubrimientos comestibles a base de almidón nativo y oxidado de yuca sobre la calidad de mango (Tommy Atkins). Temas agrarios, 18(2), 94-105.

García C., Posligua E., Mantuano L., Basurto M., Montes G., y Delgado L. (2017). Recubrimiento comestible de quitosano, almidón de yuca y aceite esencial de canela para conservar pera (Pyrus communis L. cv. "Bosc").

Gonzáles, L., Aylas, B., Paima, K., y Bazán, A. (2013). Determinación de la tasa respiratoria de Eugenia stipitata Mc Vaugh (arazá) y Myrcyaria dubia HBK (camu-camu). Ciencia Amazónica (Iquitos), 3(1), 53-58.

Gómez, E. (2011). Recubrimientos para frutas y hortalizas. V curso internacional tecnología poscosecha y procesamiento mínimo, 32.

Guerreros E., M. U. (2017). Quitosano y almidón como recubrimiento biodegradable para prolongar la vida útil en palta (Persea americana Mill.) cultivar fuerte, Arequipa-Perú.

Guillermo A., José B., Marcela V. (2013). Determinación física y bromatológica de la guanábana cimarrona (Annona glabra L.) del departamento de córdoba.

Gilbert R., Elza A., Beatriz S., Soledad Q. (2013). Efecto de las metodologías de liofilización en la calidad de frutas deshidratadas. Perú

Han, C., Lederer, C., McDaniel, M. y Zhao, Y. (2004). Sensory evaluation of fresh strawberries (Fragaria ananassa) coated with chitosan based edible coatings. Journal of FoodScience, 70, S172-8.

Hernández L., Catalina B., y Mosquera S. (2011) Efecto del recubrimiento a base de almidón de yuca modificado sobre la maduración del tomate. Revista de Investigación. Antioquia Colombia.

Hernández, N. (2013). “Caracterización fisicoquímica y microbiológica del tomate margariteño (Licopersicum esculentum var. España) y evaluación de la efectividad de tratamientos de pre-envasado para el incremento de suvida comercial a temperatura ambiente ". Universidad de Córdoba.

Hernández, K. (2016). Formulación y caracterización de películas comestibles de almidón de papa nativo y modificado. México.

Infoagro. (2010). El cultivo de la manzana. Obtenido de INFOAGRO: http://www.infoagro.com/frutas/frutas_tradicionales/manzana.htm

Javier Núñez. (2012). Evaluación de distintas técnicas de conservación en peras "Packham's Triumoh" mínimamente procesadas. Santiago de Chile.

Kader, A. (2007). Tecnología Postcosecha de Cultivo Hortofrutícolas. Centro de información e investigación en ecnologia postcosecha. California. 
Kester J., y Fennema O. (1986). Endoble films and coating: a review.

Márquez, R. (2015). Aprovechamiento tecnológico de la cera de abeja para la obtención de productos sintéticos orgánicos, no tóxicos para el ser humano. Venezuela.

Márquez M., Ramos L., y Pérez G. (2007). Efecto de la aplicación de un recubrimiento comestible a base de gelatina sobre la calidad de fresa (fragaria vesca I.) almacenando en refrigeración. México.

Márquez J., Yepes D., Sánchez L., y Osorio A. (2017). Cambios fisicoquímicos del aguacate (Persea americana Mill. cv. "Hass") en postcosecha para dos municipios de Antioquia.

Maccapa, P. (2015). Efecto de la aplicación de un recubrimiento biodegradable a base de aislado de proteína de suero sobre la vida útil de la chirimoya (annona cherimola), fresa (fragaria vesca) y pera (pyrus communis). Puno - Perú.

Medina Moral., Vicens Otero., Herrarte Sánchez. (2005). Análisis de varianza (ANOVA).

Miranda D., Armando Alvis., y Guillermo A. (2014). Efecto de dos recubrimientos sobre la calidad de la papaya (Carica papaya) variedad tainung.

Montalvo C., López A., Paulo E. (2012). Películas comestibles de proteínas: características, propiedades y aplicaciones. En Temas Selectos de Ingeniería de Alimentos (págs. 32-46). México.

Moreno L., Flores L. (2017). Evaluación del efecto de un recubrimiento a base de sábila (Aloe vera) y aceite esencial de canela (Cannamomm verum) en el tiempo de vida útil del tomate (lycopersicum esculentum Mill). Roma.

Paredes, H. (2011). Estudio de la influencia de los grados brix del chaguar mishque para la obtención de una bebida carbonatada tipo champagne. Ambato - Ecuador.

Ojeda, F. (2012). Efecto de la inclusión de nuez común (Junglas regie L.) y transglutaminasa en la elaboración de un reestructurado de carne de alpaca (Vicugna paco L.). Lima - Perú.

Parra C., Eugenio H., y Hernán C. (2006). Estudio de algunas propiedades físicas y fisiológicas precosecha de la pera variedad triunfo de Viena. Revista Bras Frutic, p. 55-59.

Parra C., y Sánchez J. (1998). Características físicas y fisiológicas de lapera variedad triunfo de Viena (Pyrus communis L). Colombia.

Pérez, M. A. A. (2009). Espacios de Color RGB, HSI y sus Generalizaciones a n-Dimensiones (Doctoral dissertation, Instituto Nacional de Astrofísica, Óptica y Electrónica). México.

Pierdrahita Gallo y Villegas Llano. (2016). Efecto de la aplicación de un recubrimiento comestible en la conservación de las características sensoriales y tiempo de almacenamiento de la mora de Castilla (Rubus glaucus Benth.) sin espinas postcosecha.

Quintero, R. (2012). Formulación de un recubrimiento comestible para frutas cítricas, estudio de su impacto mediante aproximación metabólica y evaluación de la calidad postcosecha. Colombia.

rodríguez, C. (2015). Efecto del tipo de recubrimiento y el tiempo de almacenamiento en la calidad de la carambola (Averrhoa carambola L.) almacenada en refrigeración. Ayacucho.

Rodríguez, R. (2017). Efecto del quitosano como revestimiento comestible en Yacon (Smallanthus sonchifollus) mínimamente procesado para aumentarsu vida útil. Cajamarca.

Rosario, V. (2006). Caracterización fisicoquímica del membrillo Japonés (Chaenomeles sp. Lindl.). Universidad de Murcia.

Ruiz D., Rodríguez S. (2014). Parámetros de calidad interna de hortalizas y frutas en la industria agroalimentaria.

López, C. (2006). Características fisicoquímicas y microbiológicas del tomate margariteño (Licopersicum esculentum var. España) y evaluación de la efectividad de tratamientos de pre-envasado para el incremento de su vida comercial a temperatura ambiente. España.

Tasayco, C. (7 de 5 de 2013). La Pera Beneficios, Importancia y Origen. Obtenido de Blog Pera: http://elizaysufrutolapera.blogspot.com/2013/05/la- taxonomia.html

Villamón D., Sapper M., Giné-Bordonaba J., Chiralt A., Palou L., Teixidó N., y Pérez-Gago M. B. 
(2018). Selección de recubrimientos comestibles para extender la vida útil de manzana y pera, (pg. 130-132).

Wills, R., Macglasson, B., Graham, D., y Joyce, D. (1998). Introducción a la fisiología y manipulación poscosecha de frutas, hortalizas y plantas ornamentales. Zaragoza: Acribia, S.A.

Vladimir V., Myrian S., y Katherine E. (2018). Estudio de la cera carnauba como compatibilizante en mezclas poli (ácido láctico) almidón de achira (Canna edulis). Revista Politécnica.

Waldir Chavez y Atilio Arata. (2009). El cultivo del peral en la provincia de Caravelí. Arequipa.

Vasquez L., y Vidal L. (2010). Caracterización y alternativa de uso de una película biodegradable de quitosano a partir de la extracción de quitina de langostino. Pimentel - Perú.

Yohana Ramos, Adan Casal y Beatriz Spengler. (2007). Estudio de la obtención de emulsificantes catiónicos a partir del aceite de coco. Toluca - México. 